\title{
Enhanced RAD21 cohesin expression confers poor prognosis and resistance to chemotherapy in high grade luminal, basal and HER2 breast cancers
}

Huiling $\mathrm{Xu}^{1,3+}{ }^{+}$, Max Yan ${ }^{2 \dagger}$, Jennifer Patra ${ }^{1}$, Rachael Natrajan ${ }^{4}$, Yuqian Yan', Sigrid Swagemakers ${ }^{5,6}$, Jonathan M Tomaszewski ${ }^{1,7}$, Sandra Verschoor ${ }^{1}$, Ewan KA Millar ${ }^{8,9,10,11}$, Peter van der Spek ${ }^{5}$, Jorge S Reis-Filho ${ }^{4}$, Robert G Ramsay' ${ }^{1}$ Sandra A O'Toole $8,12,13,14$, Catriona M McNeil ${ }^{8,15,16,17}$, Robert L Sutherland ${ }^{8,12}$, Michael J McKay ${ }^{1,18^{*}}$ and Stephen B Fox ${ }^{2 *}$

\begin{abstract}
Introduction: RAD21 is a component of the cohesin complex, which is essential for chromosome segregation and error-free DNA repair. We assessed its prognostic and predictive power in a cohort of in situ and invasive breast cancers, and its effect on chemosensitivity in vitro.

Methods: RAD21 immunohistochemistry was performed on 345 invasive and 60 pure in situ carcinomas. Integrated genomic and transcriptomic analyses were performed on a further 48 grade 3 invasive cancers. Chemosensitivity was assessed in breast cancer cell lines with an engineered spectrum of RAD21 expression.

Results: RAD21 expression correlated with early relapse in all patients (hazard ratio (HR) 1.74, 95\% confidence interval (Cl) 1.06 to 2.86, $P=0.029$ ). This was due to the effect of grade 3 tumors (but not grade 1 or 2 ) in which RAD21 expression correlated with early relapse in luminal $(P=0.040)$, basal $(P=0.018)$ and HER2 $(P=0.039)$ groups. In patients treated with chemotherapy, RAD21 expression was associated with shorter overall survival $(P=$ 0.020). RAD21 mRNA expression correlated with DNA copy number, with amplification present in $32 \%(7 / 22)$ of luminal, 31\% (4/13) of basal and 22\% (2/9) of HER2 grade 3 cancers. Variations in RAD21 mRNA expression in the clinical samples were reflected in the gene expression data from 36 breast cancer cell lines. Knockdown of RAD21 in the MDA-MB-231 breast cancer cell line significantly enhanced sensitivity to cyclophosphamide, 5-fluorouracil and etoposide. The findings for the former two drugs recapitulated the clinical findings.
\end{abstract}

Conclusions: RAD21 expression confers poor prognosis and resistance to chemotherapy in high grade luminal, basal and HER2 breast cancers. RAD21 may be a novel therapeutic target.

\section{Introduction}

Cohesin is a multi-protein complex that is highly conserved from yeast to humans. Its primary role is to adhere sister chromatids in close apposition, a mechanism termed 'sister chromatid cohesion' (SCC). SCC is

\footnotetext{
* Correspondence: Michael.McKay@act.gov.au; stephen.fox@petermac.org

+ Contributed equally

'Research Division, Peter MacCallum Cancer Centre, Locked Bag 1, A'Beckett Street, Melbourne, Vic 8006, Australia

2Department of Pathology, Peter MacCallum Cancer Centre, Locked Bag 1,

A'Beckett Street, Melbourne, Vic 8006, Australia

Full list of author information is available at the end of the article
}

fundamental to several key cellular processes, including chromosome segregation during mitosis and meiosis, error-free homologous recombinational repair (HRR) of DNA double strand breaks and the regulation of gene transcription [1-7]. The core cohesin complex consists of four proteins, RAD21 (also known as SCC1 or MCD1), SMC1, SMC3 and SCC3 [8].

RAD21 is a central component of the cohesin complex, both structurally and functionally [8]. Aberrant $R A D 21$ expression has been reported in multiple cancers and cancer cell lines [9-12]. In a mega-scale microarray

\section{Biomed Central}


analysis of multiple cancers, $R A D 21$ was identified as one of 69 signature genes in undifferentiated cancers that had aggressive in vitro or clinical courses and poor patient outcomes [9]. Further, an intronal single nucleotide polymorphism (SNP) in the RAD21 gene is strongly associated with increased breast cancer risk [10]. Although these reports support the notion that the abnormal activity of RAD21 may be an important feature of human breast cancer, there are no data available from clinical breast cancer samples.

We therefore, evaluated RAD21 expression in a cohort of well-characterised human in situ and invasive breast cancers to 1) assess the correlation between RAD21 expression, and conventional and molecular clinicopathological parameters and patient prognostic data; and 2) determine whether aberrant RAD21 expression might predict therapeutic outcomes.

\section{Materials and methods}

\section{Patient clinicopathological variables}

The flow of patients through the study according to the reporting recommendations for tumor marker prognostic studies (REMARK) criteria [13] is listed in Additional file 1 . Four hundred and nine invasive cancers were obtained from the Garvan Institute (292 cases with survival and treatment data) and the Peter MacCallum Cancer Centre (117 cases without survival data). Sixtyfour cases were excluded due to lack of tissue available for tissue microarray (TMA) construction or absence of tumor on the array. The final cohort of invasive cancers was 345 cases (251 cases with survival data). For ductal carcinoma in situ (DCIS), 60 cases of pure DCIS were obtained from the John Radcliffe Hospital, UK, and were assessed on TMAs. This study has Ethics Committee approvals (numbers 00/81, 03/90, 09/36, JRC02.216, HREC SVH H94/080 and HREC SVH 06336 H00036). Patient median age was 54 years (range 24 to 87 years). Forty-one percent of patients received adjuvant chemotherapy with cyclophosphamide/methotrexate/5fluorouracil (CMF), or doxorubicin (adriamycin)/cyclophosphamide (AC). Fifty-two percent received adjuvant endocrine therapy with tamoxifen. Patient median follow-up was 58.1 months. During this time, 100 patients developed recurrence (24.4\%) and 86 deaths $(21.0 \%)$ were considered breast-cancer related.

\section{Immunohistochemistry}

TMAs were constructed from $1 \mathrm{~mm}$ diameter (invasive cancers) or $2 \mathrm{~mm}$ cores (DCIS). Sections of $4 \mu \mathrm{m}$ thickness were used for immunostaining using the EnVision ${ }^{\mathrm{TM}}$ kit (DAKO, Glostrup, Denmark) following the manufacturer's instructions. Briefly, de-paraffinized and rehydrated tissue sections were treated for antigen retrieval in $10 \mathrm{mM}$ Tris- $\mathrm{HCl}$ ( $\mathrm{pH}$ 9.0) buffer containing
$1 \mathrm{mM}$ EDTA for three minutes at $125^{\circ} \mathrm{C}$ in a pressure cooker (Biocare Decloker, Concord, CA, USA). Sections were then treated with $3 \% \mathrm{H}_{2} \mathrm{O}_{2}$ for five minutes to remove endogenous peroxides, washed and incubated with a rabbit polyclonal anti-RAD21 antibody (1:200) (Abcam, Cambridge, UK), with anti-rabbit IgG as negative control, for two hours at room temperature. Horseradish peroxidase (HRP)-conjugated secondary antibody, the signal was detected using DAB (3', 3'-diaminobenzidine) substrate. Sections were counter-stained with hematoxylin to visualize nuclei. Validation of the antiRAD21 antibody was performed using small interference RNA (siRNA) knockdown of the human RAD21 gene in MCF10A cells on cell blocks (Additional file 2).

Nuclear RAD21 expression was assessed for intensity $(0$ = no staining, 1 = weak, 2 = moderate, $3=$ strong) and the percentage of positive cells $(0=0 \%, 1 \leq 10 \%, 2=10 \%$ to $50 \%, 3=51 \%$ to $80 \%, 4 \geq 80 \%$ positive cells) as defined previously [14]. The scores for intensity and percentage were added and a cut-off of 7 was used to define two approximately equal size groups of patients for subsequent statistical analyses.

ER, HER2, EGFR and CK5/6 staining were used to classify tumors into four intrinsic subgroups: the basal group (ER negative, HER2 negative, CK5/6 and/or EGFR positive), luminal group (ER positive, HER2 negative), HER2 group (HER2 positive) and the negative (null) group (ER, HER2, CK5/6 and EGFR negative) [15].

\section{Cell lines}

Cell lines (MCF7, MCF10A, MDA-MB-231, MDA-MB468, SK-BR-3, T47 D and ZR75-1) were obtained from the American Type Culture Collection (ATCC, Rockville, MD, USA). MCF10A was grown and maintained in a 1:1 mixture of Dulbecco's modified Eagle medium (DMEM) F-12 supplemented with $5 \%$ horse serum, $20 \mathrm{ng}$ of epidermal growth factor (EGF) per $\mathrm{ml}, 10 \mu \mathrm{g} / \mathrm{ml}$ of insulin, and 0.5 $\mu \mathrm{g} / \mathrm{ml}$ of hydrocortisone. MDA-MB-468, SK-BR-3 and ZR75-1 were grown in MEM with $10 \%$ fetal bovine serum (FBS) and 1\% Pen/Strep. Other cell lines were grown in RPMI-1640 with 10\% FBS and 1\% Pen/Strep. SVCT was obtained from European Collection of Cell Cultures (ECACC; Salisbury, Wilts, UK) and grown in DMEM with $10 \% \mathrm{FBS}, 5 \mu \mathrm{g} / \mathrm{ml}$ hydrocortisone, and $10 \mu \mathrm{g} / \mathrm{ml}$ insulin.

\section{Quantitative real time PCR and Semi-quantitative Western} blot analysis

Exponentially growing cells were harvested and total RNA was extracted using RNeasy kits (Qiagen, Valencia, CA, USA). DNA was removed by on-column treatment with RNase-free DNase. One microgram of total RNA was used for cDNA synthesis using a Superscript III kit (Invitrogen, Carlsbad, CA, USA). cDNA was used for quantitative real time polymerase chain reaction (qRTPCR) using primers (5'-AATTTGGCTAGCGGCCCAT3' and 5'-TGTCCGTAATGCCATTTTCACC-3') which 
span exon 10 and exon 11 of the human RAD21 gene. Phosphoglycerate kinase (PGK) was used as a housekeeping gene and the relative expression of RAD21 gene was determined from three independent experiments using the DeltaDelta CT method [16].

Total protein extraction and Western blots were carried out essentially as described previously [17]. For Western blot analysis, blots were incubated with a rabbit polyclonal anti-RAD21 antibody (Abcam, Cambridge, UK) followed by a fluorescence-conjugated secondary antibody, Alexa 680 anti-rabbit (Invitrogen, Carlsbad, CA, USA). The signal intensity was measured using the Li-Cor Odyssey system. Membranes were then probed with a mouse monoclonal anti-pan actin antibody (Cell Signaling Technology, Danvers, MA, USA) followed by an IRDye800conjugated anti-mouse antibody (Rockland, Gilbertsville, PA, USA). The relative level of protein expression was determined by normalizing to the pan-actin loading control from a minimum of three independent experiments.

\section{Array CGH and gene expression analysis}

Array comparative genomic hybridization (CGH) and microarray-based expression profiling were obtained from analysis of 48 microdissected grade 3 invasive ductal carcinomas as described [18]. Tumor subtypes were determined as described above [15].

\section{Gene expression data mining}

Gene expression profiles of 38 breast cancer cell lines were obtained by mining a microarray dataset described by Hollestelle et al. (2009) [19] at GEO (accession number (GEO: GSE16795)) [20]. Raw intensity values of all samples were normalized by RMA normalization (Robust Multichip Analysis) (background correction and normalization) using Partek version 6.4 (Partek Inc., St. Louis, MO). The normalized data file was transposed, back transformed to normal intensity values and imported into OmniViz version 6.0.1 (BioWisdom Ltd., Cambridge, UK) for further analysis. For each probe set, the geometric mean of the hybridization intensity of all samples was calculated. The level of expression of each probe set was determined relative to this geometric mean and ${ }^{2} \log$-transformed. The geometric mean of the hybridization signal of all samples was used to ascribe equal weight to gene expression levels with similar relative distances to the geometric mean. Next a query-by-example numerical query was performed in OmniViz to find records most closely related to RAD21. The top 25 genes that correlated the best with $R A D 21$ were visualized using a treescape view.

\section{Generation of stable RAD21 knockdown cell lines and clonogenic survival assays}

A single cell clone was derived from the human breast cancer cell line, MDA-MB-231. This cell line is of the basal subtype. Two different short-hairpin RNA (shRNA) shRNAmir constructs, shRNA 57223 and shRNA 57224, and shRNAmir vector control (Open Biosystems, Huntsville, AL, USA) were introduced into cells using Lipofectamine 2000 (Invitrogen, Carlsbad, CA, USA) and clones were selected following a two-week culture in $2 \mu \mathrm{g} / \mathrm{ml}$ puromycin. Work involving recombinant DNA was conducted in approved Physical Containment level 2 (PC2) facilities. Western blot analysis and quantitative real-time PCR were used to verify the level of RAD21 protein and mRNA.

Clonogenic survival was performed as described [21]. Exponentially growing cells were seeded in triplicate plates, allowed to adhere for four to six hours. Following 24-hour incubation with individual drugs at graded concentrations, cells were cultured for two weeks. Surviving colonies defined as containing more than 50 cells were counted. Three independent experiments were performed for each treatment. Survival curves were generated using the linear quadratic model, GraphPad Prism version 5.01 for Windows, GraphPad Software, San Diego, CA, USA.

\section{Statistical analysis}

Correlations were examined using the one-way ANOVA, Students $t$-test, or chi-square test where appropriate. Kaplan-Meier survival curves were calculated using tumor recurrence (relapse free survival) and breast cancer-related death (overall survival) as the endpoints and compared using a log rank test. Binary logistic regression was used for multivariate analyses and the Cox proportional hazard regression model was used to identify independent prognostic factors for disease-free and overall survival. Analyses were performed with SPSS 16.0 (SPSS Inc., IL, USA). A two-tailed $P$-value test was used in all analyses and a $P$-value of less than 0.05 was considered statistically significant.

\section{Results}

\section{RAD21 protein expression in in situ and invasive breast} cancer

Expression of RAD21 in DCIS ranged from negative $(2 / 60$ cases, $3 \%)$ to heterogenous staining (30/60 cases, $50 \%)$ and homogenous strong staining (28/60 cases, 47\%) (Figure 1A). For invasive cancers, RAD21 staining patterns were similar to DCIS, ranging from heterogeneous (50/95 cases, $53 \%)$ and homogeneous strong staining $(42 / 95$ cases, $44 \%)$ to negative (3/95 cases, $3 \%)$ (Figure 1B, C). No staining or minimal weak staining was present in the cytoplasm in all cases. Expression of RAD21 in invasive cancers was significantly lower $(104 / 343,30 \%)$ than in in situ cancers $(28 / 60,47 \%)$ $(P=0.001)$. 
Table 1 RAD21 expression by DCIS grade $(P=\mathbf{0 . 4 2 8})$

\begin{tabular}{lccc}
\hline Grade & $\begin{array}{c}\text { Negative } \\
\boldsymbol{n}(\%)\end{array}$ & $\begin{array}{c}\text { Positive } \\
\boldsymbol{n}(\%)\end{array}$ & $\begin{array}{c}\text { Total } \\
\boldsymbol{n}(\%)\end{array}$ \\
\hline Low & $3(50.0 \%)$ & $3(50.0 \%)$ & $6(100.0 \%)$ \\
Intermediate & $9(60.0 \%)$ & $6(40.0 \%)$ & $15(100.0 \%)$ \\
High & $8(38.1 \%)$ & $13(61.9 \%)$ & $21(100.0 \%)$ \\
\hline Total & $\mathbf{2 0 ( 4 7 . 6 \% )}$ & $\mathbf{2 2 ( 5 2 . 4 \% )}$ & $\mathbf{4 2 ( 1 0 0 . 0 \% )}$ \\
\hline
\end{tabular}

DCIS, ductal carcinoma in situ.

but not with tumor grade $(P=0.328)$, age $(P=0.815)$, HER2 status $(P=0.564)$ or ER status $(P=0.054)$ (Table 3). Positive RAD21 expression was seen in $37 \%$ (75/201) luminal, 24\% (10/42) basal, 22\% (9/49) HER2 and $18 \%(5 / 28)$ null, cancers. When compared to luminal cancers, null type cancers, but not basal and HER2 cancers, were significantly more likely to be RAD21 negative $(P=0.043)$ (Table 4$)$.

\section{RAD21 expression and its correlation with relapse-free survival}

There was a significant correlation between positive RAD21 expression and shorter relapse-free survival (RFS) $(P=0.009)$ (Figure $2 \mathrm{~A})$. The association with early relapse was confirmed on multivariate analysis $(P=0.029, \mathrm{HR}=$ $1.74,95 \%$ CI 1.06 to 2.86) (Table 5). Subset analysis revealed RAD21 expression correlated with relapse in grade $3(P=0.023)$ (Figure $2 \mathrm{~B})$ but not in grade 1 or 2 tumors $(P=0.342)$ (Figure $2 C)$. Further analysis of grade 3 tumors according to subtype showed a significant correlation between RAD21 expression and shorter RFS in the grade 3 luminal $(P=0.040)$ (Figure $2 D)$, grade 3 basal $(P=$ 0.018 ) (Figure $2 \mathrm{E})$ and grade 3 HER2 cancers $(P=0.039)$ (Figure $2 \mathrm{~F})$, but not null type cancers $(P=0.247)$.

RAD21 expression and its correlation with overall survival in patients treated with chemotherapy

Among patients not treated with chemotherapy, there was no correlation between RAD21 expression and overall survival $(P=0.779)$ (Figure $2 G)$, whereas among patients treated with chemotherapy there was a significantly shorter overall survival in patients whose tumors were positive for RAD21 expression $(P=0.020)$ (Figure $2 \mathrm{H}$ ). This association is also true for patients with grade 3

\section{clinicopathological characteristics in DCIS}

RAD21 expression did not significantly associate with nuclear grade $(P=0.428)$ (Table 1$)$ or the intrinsic phenotypes in DCIS $(P=0.471)$ (Table 2$)$. There was no correlation between RAD21 expression in DCIS and risk of relapse $(P=0.834)$.

\section{Association between RAD21 expression and clinicopathological characteristics and intrinsic subtypes in invasive cancer}

RAD21 expression correlated with larger tumor size $(P=0.012)$ and lymph node involvement $(P<0.001)$,

\begin{tabular}{|c|c|c|c|}
\hline Subtype & $\begin{array}{c}\text { Negative } \\
n(\%)\end{array}$ & $\begin{array}{c}\text { Positive } \\
n(\%)\end{array}$ & $\begin{array}{l}\text { Total } \\
n(\%)\end{array}$ \\
\hline Luminal & $21(55.3 \%)$ & $17(44.7 \%)$ & $38(100 \%)$ \\
\hline Basal & $0(.0 \%)$ & 1 (100.0\%) & 1 (100\%) \\
\hline HER2 & 7 (43.8\%) & 9 (56.2\%) & 16 (100\%) \\
\hline Null & 3 (75.0\%) & 1 (25.0\%) & $4(100 \%)$ \\
\hline Total & 31 (52.5\%) & $28(47.5 \%)$ & $59(100.0 \%)$ \\
\hline
\end{tabular}

DCIS, ductal carcinoma in situ. 
Table 3 RAD21 expression in invasive carcinoma by clinicopathological parameters

\begin{tabular}{|c|c|c|c|c|}
\hline & $\begin{array}{c}\text { negative } \\
n(\%)\end{array}$ & $\begin{array}{c}\text { positive } \\
n(\%)\end{array}$ & $\begin{array}{l}\text { Total } \\
n(\%)\end{array}$ & $P$ value \\
\hline Grade & & & & $P=0.328$ \\
\hline 1 & $42(17.6 \%)$ & $16(15.4 \%)$ & $58(16.9 \%)$ & \\
\hline 2 & $96(40.2 \%)$ & 35 (33.7\%) & $131(38.2 \%)$ & \\
\hline 3 & $101(42.3 \%)$ & $53(51.0 \%)$ & $154(44.9 \%)$ & \\
\hline Size & & & & $P=0.012$ \\
\hline$<20 \mathrm{~mm}$ & $147(61.8 \%)$ & $50(47.2)$ & $197(57.3 \%)$ & \\
\hline$>20 \mathrm{~mm}$ & 91 (38.2\%) & $56(52.8 \%)$ & $147(42.7)$ & \\
\hline Lymph node & & & & $P<0.001$ \\
\hline Negative & 148 (63.5\%) & $43(42.2 \%)$ & $191(57.0 \%)$ & \\
\hline Positive & 85 (36.5\%) & $59(57.8 \%)$ & $144(43.0 \%)$ & \\
\hline Age & & & & $P=0.815$ \\
\hline$<50$ & 84 (35.1\%) & 39 (36.4\%) & $123(35.5 \%)$ & \\
\hline$>50$ & $155(64.9)$ & 68 (63.6\%) & $223(64.5 \%)$ & \\
\hline ER & & & & $P=0.054$ \\
\hline Negative & 63 (34.4\%) & $14(21.5 \%)$ & 77 (31/0\%) & \\
\hline Positive & $120(65.6 \%)$ & 51 (78.5\%) & $171(69.0 \%)$ & \\
\hline HER2 & & & & $P=0.564$ \\
\hline Negative & 142 (79.8\%) & $54(83.1 \%)$ & $196(80.7 \%)$ & \\
\hline Positive & $36(20.2 \%)$ & 11 (16.9\%) & 47 (19.3\%) & \\
\hline Chemotherapy* & & & & $P=0.032$ \\
\hline No & 117 (62.95) & $31(47.7 \%)$ & $148(59.0 \%)$ & \\
\hline Yes & 69 (37.1\%) & $34(52.3 \%)$ & $103(41.0 \%)$ & \\
\hline Endocrine therapy & & & & $P=0.034$ \\
\hline No & 97 (52.2\%) & $24(36.9 \%)$ & $121(48.2 \%)$ & \\
\hline Yes & 89 (47.8\%) & $41(63.1 \%)$ & $130(51.8 \%)$ & \\
\hline
\end{tabular}

* Chemotherapy was given either as cylcophosphamide/methotrexate/ fluorouracil (CMF), or doxorubicin (adriamycin)/cyclophosphamide (AC).

$E R$, oestrogen receptor.

tumors $(P=0.021)$. No significant difference in overall survival was seen in patients treated with endocrine therapy, when stratified by RAD21 expression $(P=0.231)$.

RAD21 gene expression correlates with copy number alterations, and RAD21 is amplified in a subset of grade 3 luminal, basal and HER2 cancers

In view of the correlation of RAD21 expression with prognosis in grade 3 cancers, we examined $R A D 21$ mRNA expression for its association with gene copy

Table 4 RAD21 expression in breast cancers by intrinsic subtype

\begin{tabular}{lcccc}
\hline Subtype & $\begin{array}{c}\text { negative } \\
\boldsymbol{n}(\%)\end{array}$ & $\begin{array}{c}\text { positive } \\
\boldsymbol{n}(\%)\end{array}$ & $\begin{array}{c}\text { Total } \\
\boldsymbol{n}(\%)\end{array}$ & $\begin{array}{c}\text { P-value } \\
\text { Relative to luminal }\end{array}$ \\
\hline Luminal & $126(63 \%)$ & $75(37 \%)$ & $201(100 \%)$ & - \\
Basal-like & $32(76 \%)$ & $10(24 \%)$ & $42(100 \%)$ & $\mathbf{0 . 0 9 5}$ \\
HER2 & $38(78 \%)$ & $11(22 \%)$ & $49(100 \%)$ & 0.050 \\
Null & $23(82 \%)$ & $5(18 \%)$ & $28(100 \%)$ & $\mathbf{0 . 0 4 3}$ \\
\hline Total & $\mathbf{2 3 9 ( 7 0 \% )}$ & $\mathbf{1 0 4 ( 3 0 \% )}$ & $\mathbf{3 2 0 ( 1 0 0 \% )}$ & \\
\hline
\end{tabular}
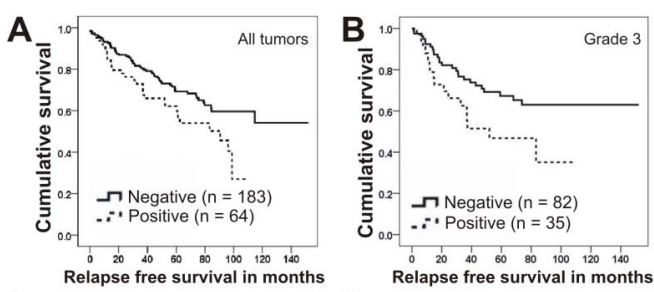

C
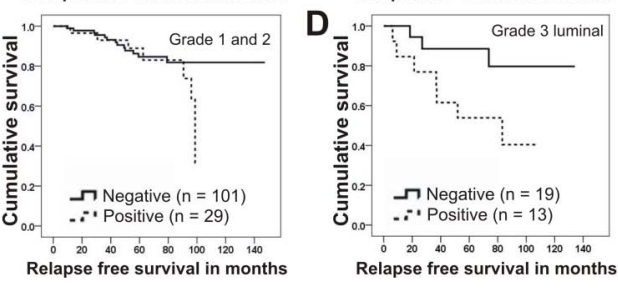

E
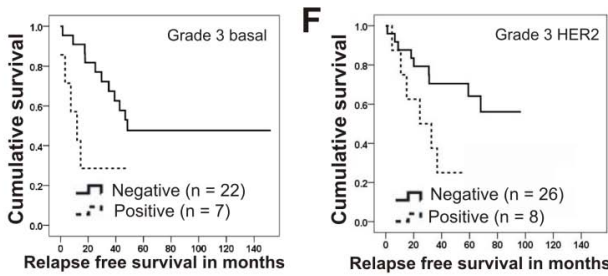

$\mathbf{G}$
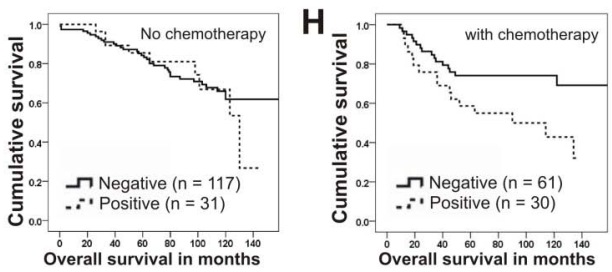

Figure 2 Kaplan-Meier curves stratified by nuclear RAD21 expression, relapse-free survival (A-F) and overall survival (GH). Relapse free survival: $A$, in all tumors, $(P=0.009)(n=247) ; B$, grade 3 cancers $(P=0.023)(n=117) ; C$, grade 1 and 2 cancers $(P=$ $0.342)(n=130)$; $D$, grade 3 luminal cancers $(P=0.040)(n=32)$; , grade 3 basal cancers $(P=0.018)(n=29) ; F$, grade 3 HER2 cancers $(n=34)$; Overall survival: $\mathrm{G}$, without chemotherapy $(P=0.779)(n=$ 148). $H$, treated with chemotherapy $(P=0.020)(n=91)$.

number, in an integrated array $\mathrm{CGH}$ and transcriptional dataset generated from 48 microdissected grade 3 invasive ductal carcinomas of luminal $(n=22)$, basal-like ( $n$ $=13)$ and HER2 $(n=13)$ subtypes [18]. Array CGH and microarray expression profiling showed RAD21 mRNA expression correlated with gene copy number in luminal

Table 5 Multivariate analysis, Cox regression model of relapse-free survival in all breast cancers

\begin{tabular}{llll}
\hline & $P$ value & Hazard Ratio & 95\% Cl for hazard ratio \\
\hline RAD21 & 0.029 & 1.74 & 1.06 to 2.86 \\
Grade & 0.018 & 1.63 & 1.09 to 2.44 \\
Size $>\mathbf{2 0} \mathbf{~ m m}$ & 0.656 & 1.11 & 0.71 to 1.73 \\
Lymph node status & 0.023 & 1.69 & 1.07 to 2.65 \\
ER & 0.003 & 0.46 & 0.28 to 0.77 \\
Age $>\mathbf{5 0}$ & 0.325 & 1.28 & 0.781 to 2.11 \\
\hline
\end{tabular}

$\mathrm{Cl}$, confidence interval; $\mathrm{ER}$, oestrogen receptor. 
$(P=0.003)$, basal $(P=0.0086)$ and HER2 $(P=0.0035)$ tumors (Pearson correlation, Table 6). RAD21 amplification is present in $32 \%(7 / 22)$ of luminal, $31 \%(4 / 13)$ of basal and $22 \%(2 / 9)$ of HER2 subtypes. These proportions were very similar to our immunohistochemistry analysis of a different sample set described above, where $30 \%$ of luminal (14/46), $25 \%$ of basal (10/40), and $22 \%$ of HER2 (9/41) grade 3 cancers showed positive RAD21 expression. Collectively, these data suggest that positive RAD21 expression observed in a subset of grade 3 tumors may be due to gene amplification.

\section{RAD21 expression in breast cancer cell lines}

Variations in RAD21 protein expression in clinical samples were reflected by gene expression analysis using qRT-PCR of a panel of breast cancer cell lines (Figure 3A), and by microarray profiling of 36 breast cancer cell lines derived from Hollestelle et al. [19] (Figure 3B). Further analysis revealed that TOP $2 A$ which encodes topoisomerase II (a protein also required for sister chromatid separation) and NIPBL (encoding a cohesin loading protein) are among top 25 genes positively correlated with $R A D 21$ expression (Figure 3B).

\section{Knockdown of RAD21 gene expression with short-hairpin RNA in a basal-like breast cancer cell line, MDA-MB-231, results in its enhanced sensitivity to chemotherapeutic drugs}

To test the functional significance of our cancer therapy results that RAD21 expression affects sensitivity to chemotherapeutic drug response, we used a small hairpin shRNA-mediated gene-silencing approach to knockdown the $R A D 21$ gene in MDA-MB-231 breast cancer cell line. Of several independent cell clones generated using two different shRNA constructs, three clones exhibited a reduction in both $R A D 21$ transcripts and protein (Figure 4). The relative levels of $R A D 21$ mRNA in four stable clones, as determined by qRT-PCR analysis, were

Table 6 Correlation of RAD21 gene expression with genomic alterations*

\begin{tabular}{lccc}
\hline Subtype & $\begin{array}{c}\text { Copy number } \\
\text { Pearson } \\
\text { correlation } \\
P \text {-value }\end{array}$ & $\begin{array}{c}\text { Gaina } \\
\text { Mann Whitney } \\
\text { U test } \\
P \text {-value }\end{array}$ & $\begin{array}{c}\text { Amplificationb } \\
\text { Mann Whitney } \\
\text { U test } \\
P \text {-value }\end{array}$ \\
\hline $\begin{array}{l}\text { Luminal }(n= \\
\begin{array}{l}\text { 22) } \\
\text { Basal-like ( } n \\
=13)\end{array}\end{array}$ & 0.0030 & 0.0169 & 0.0465 \\
HER2 $(n=13)$ & 0.0086 & - & 0.0111 \\
\hline
\end{tabular}

* Patients and Methods were as described in Natrajan et al. (2009).

${ }^{\text {a }}$ Gain corresponds to approximately three to five copies of the locus as defined using a smoothed Log2 ratio of between 0.08 and 0.45 .

${ }^{b}$ Amplification was defined as having a Log2 ratio $>0.45$, corresponding to more than five copies.

$\mathrm{CGH}$, comparative genomic hybridization.

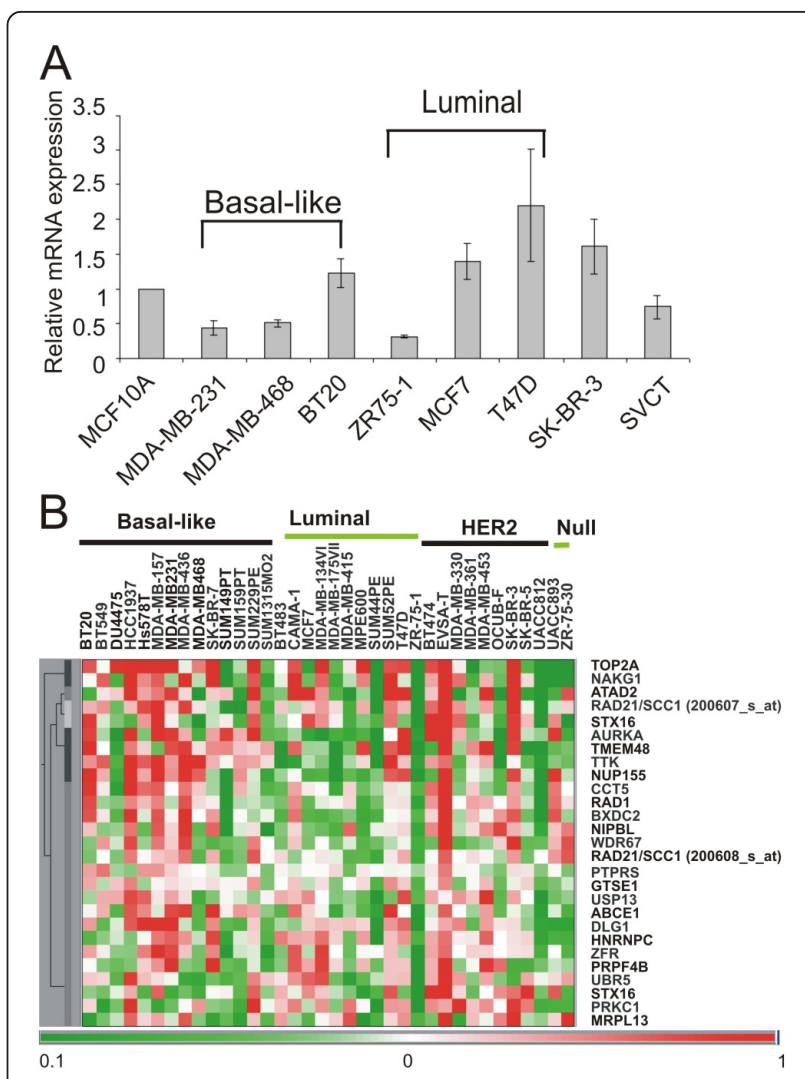

Figure 3 Expression of RAD21 in breast cancer cell lines. A. Quantitative real time RT-PCR of RAD21 transcripts in human breast cancer cell lines. The expression level in MCF10A was used as a reference and given an arbitary value of 1 . Relative expression of RAD21 gene was compared with MCF10A. Error bar = standard error mean (SEM). B. OmniViz Treescape showing the hierarchical clustering of the top 25 genes that correlated best with the two RAD21 gene probes (200607_s_at and 200608_s_at). Gene expression levels: red, up-regulation compared with the geometric mean; green, down-regulation compared with the geometric mean. The color intensity correlates with the degree of change. Raw dataset (GEO:GSE16795) was sourced from GEO [20].

$56 \pm 4 \%$ for sh223_sc1 $(P=0.010), 90 \pm 13 \%$ for sh223_sc3 $(P=0.531), 62 \pm 13 \%$ for sh224_sc4 $(P=$ $0.111)$ and $55 \pm 2 \%$ for sh224_sc5 $(P=0.002)$, relative to the parental cell line (Figure $4 \mathrm{~A}$ ). No apparent reduction in RAD21 mRNA was detected in the control clone transfected with shRNAmir vector $(96 \% \pm 8 \%, P=$ 0.726) (Figure 4A). Further examination of the corresponding RAD21 protein by semi-quantitative Western blot analysis revealed a statistically significant reduction in the levels of RAD21 protein in the three clones (sh223_sc1, sh224_sc4 and sh224_sc5), which exhibited RAD21 mRNA reduction (Figure 4A, B). The relative levels of RAD21 protein were $82 \% \pm 4 \%$ for sh223_sc1 $(P=0.017), 65 \pm 4 \%$ for sh224_sc4 $(P=0.002)$ and $60 \pm$ $6 \%$ for sh224_sc5 $(P=0.007)$, relative to the parental cell line (Figure 4B). The RAD21 protein levels in the 


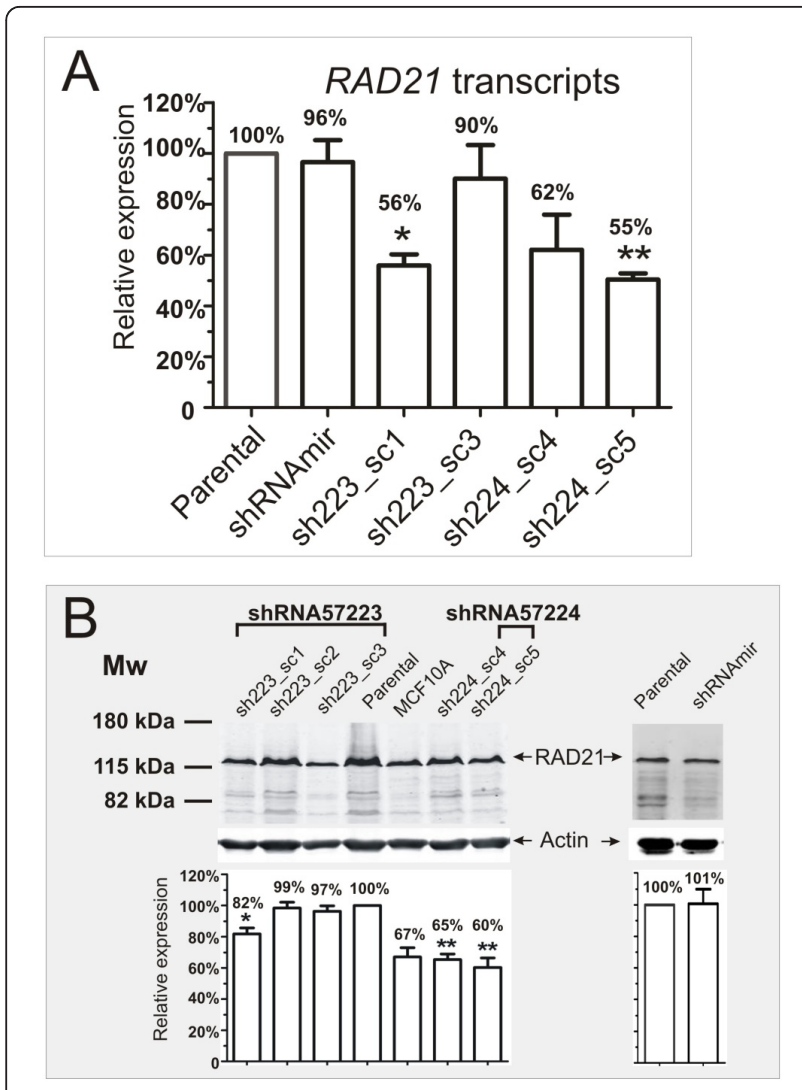

Figure 4 Validation of RAD21 expression in MDA-MB-231 cells carrying shRAD21 knockdown constructs. A. Quantitative real time PCR analysis of RAD21 expression in clones stably expressing two different shRAD21 constructs and shRNAmir vector, relative to the parental cell line. B. Western blot analysis of RAD21 protein level. RAD21 expression in five independently derived clonal cell lines with two different stable RAD21 knockdown constructs was compared to the parental cell line, cells transfected with shRNAmir vector and an immortalized human breast epithelial cell line MCF10A. Pan-actin was used as loading control. A reduction in the levels of RAD21 protein in three stable clones (sh223_sc1, sh224_sc4 and sh224_sc5) was verified by semi-quantitative Western blot analysis (bottom panel). The levels of RAD21 protein were

normalized to the pan-actin loading control and expressed as the percentage of the parental line where the RAD21 expression was given an arbitrary value of $100 \%$. The values represent the mean of four independent experiments except for shRNAmir vector and MCF10A where three independent experiments were performed.

Clones with a significant reduction in either RAD21 mRNA or protein levels were marked by asterisks $\left({ }^{*} P<0.05\right.$; ${ }^{* *} P<0.005$, Student $t$ test). Error bar $=$ SEM.

sh224_sc4 and sh224_sc5 clones are comparable to that in an immortalized human mammary epithelial cell line, MCF10A (Figure 4B). No apparent reduction in RAD21 protein was detected in sh223_sc2 $(99 \% \pm 4 \%, P=$ 0.700), sh223_sc3 (97 $\pm 4 \%, P=0.367)$ and shRNAmir vector $(101 \pm 9 \%, P=0.947)$, compared to the parental line (Figure 4B). Thus, reduction of both RAD21 mRNA and protein levels was confirmed in three stable shRAD21 knockdown clones.

We next tested the response of shRAD21 clones to two breast cancer chemotherapeutic drugs, cyclophosphamide and 5-fluorouracil (5-FU). All three RAD21 knockdown clones, sh223_sc1, sh224_sc4 and sh224_sc5, showed increased sensitivity to the drug following treatment with cyclophosphamide, in a manner that directly correlates with the level of RAD21 expression (Figure 5A). In contrast, such enhanced sensitivity was not observed in the clone sh223_sc3 which did not show a reduction in either $R A D 21$ mRNA or protein level (Figure 4). We noted that the sh223_sc1 clone exhibited a more reduced clonogenic survival compared to the other clones, sh224_sc4 and sh224_sc5. This may be due to the difference in targeting sequences between the sh223 and sh224 shRNAs. Similarly, treatment of three shRAD21 clones (sh223_sc1, sh224_sc4 and sh224_sc5) with 5-FU resulted in a significant reduction in the clonogenic survival of all three clones compared to the parental line (Figure $5 \mathrm{~B}$ ). These data recapitulate our findings in patients treated with cyclophosphamide/ methotrexate/5-fluorouracil (CMF) or doxorubicin (adriamycin)/cyclophosphamide (AC) (Figure 2H), providing further evidence that RAD21 expression correlates with cellular sensitivity to chemotherapeutic-drugs.

Since our analysis showed that RAD21 expression strongly correlates with $T O P 2 A$ expression in a number of breast cancer cell lines (see Figure 3), we assessed the sensitivity of $R A D 21$ knockdown clones to etoposide, a topoisomerase II inhibitor and commonly used anti-cancer drug. Etoposide treatment also led to a marked decrease in cell survival in the shRAD21 clones tested, compared to the parental cells (Figure 5C). This result is consistent with an early report of an enhanced etoposide-sensitivity following a siRNA-mediated transient RAD21 knockdown in MCF7 breast cancer cell line [10].

\section{Discussion}

This translational study is the first comprehensive analysis of a novel chromosomal DNA repair protein, RAD21 cohesin, in breast cancer. Our analyses provide compelling evidence that RAD21 expression is a novel prognostic marker in breast cancer and is also highly predictive of anti-cancer therapy outcomes. Tumor RAD21 overexpression strongly correlated with amplification of the $R A D 21$ gene locus in a significant subset of high grade luminal, basal and HER2 cancers. This suggests that the observed RAD21 overexpression resulted from gene amplification, and provides a plausible explanation for the strong RAD21 prognostic effects observed in these tumors. Our findings in breast cancer with the RAD21 cohesin may also generalize to some other epithelial 


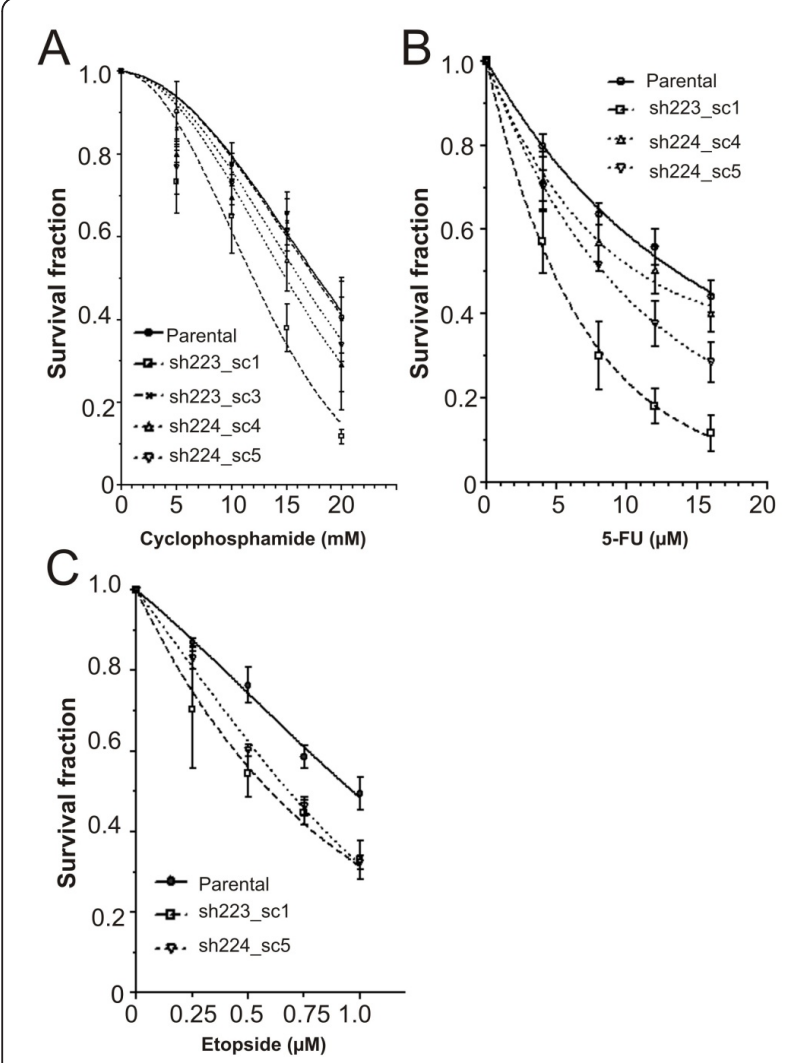

Figure 5 Effect of shRNA-mediated RAD21 knockdown on cellular sensitivity to anti-cancer drugs. Independent cell clones with stably reduced $R A D 21$ expression were derived from the breast cancer cell line, MDA-MB-231. Relative levels of RAD21 gene expression when compared to the parental line by qRT-PCR and semi-quantitative Western blot analysis were shown in Figure 4. Error bar $=$ SEM. Clonogenic survival following treatment with: $\mathbf{A}$ cyclophosphamide: sh223_sc1 $(P=0.0316)$, sh223_sc3 $(P=0.175)$, sh224_sc4 $(P=0.0187)$ and sh224_sc5 $(P=0.0563) ;$ B. 5-FU: sh223_scl $(P=0.020)$, sh224_sc4 $(P=0.0257)$ and sh224_sc5 $(P=$ $0.0242)$; and $C$. etoposide: sh223_sc1 $(P=0.020)$ and $\operatorname{sh} 224 \_s c 5(P=$ 0.042).

cancer types: consistent with our data, RAD21 at chromosomal locus $8 \mathrm{q} 24$ is also commonly amplified in advanced androgen-resistant prostate cancer [11].

Our immunohistochemical analysis showed that RAD21 expression associates with shorter relapse-free survival in patients with high grade breast cancer. Based on known RAD21 functions, the adverse outcome in breast cancer patients with RAD21 expression could be due to an elevated level of homologous recombination (HR) repair activity as a result of RAD21 overexpression. We favor this explanation. Overexpression of other HR proteins (for example, RAD51, BRCA1) has also been shown to be associated with increased resistance to radio- and chemo-therapy $[22,23]$. Furthermore, should RAD21 expression prove to be a surrogate for HR activity, this may provide a simple, cost-effective and novel way for evaluating $\mathrm{HR}$ activity on tissue sections. Alternatively, numerical chromosome (ploidy) alterations as a result of chromosome segregation errors could contribute to the poorer survival outcomes we observed.

RAD21 expression is a prognostic and predictive factor that affects the ultimate outcome in many breast cancer sub-histotypes. Prior to clinical use, these data will require validation, for example, in another (larger) clinical dataset, including assessment in a randomised clinical trial. Once validated, RAD21 expression would have potential translational use in the clinical management of patients. Immunohistochemical evaluation of RAD21 protein on breast cancer specimens could be routinely incorporated into standard pathology reporting to estimate levels of RAD21 that might determine drug response of certain drug regimens.

Furthermore, our survival analysis revealed that in patients receiving chemotherapy, those patients with tumors positive for RAD21 expression showed a significantly shorter overall survival than patients whose tumors were negative for RAD21, highlighting an exciting potential role for RAD21 expression in predicting cancer therapy response. We further verified the relevance of RAD21 expression to the therapeutic response in vitro. Stable knockdown of RAD21 significantly enhanced, in a graded fashion, cellular sensitivity to 5-FU, cyclophosphamide and etoposide. The first two of these drugs are components of the commonly used FEC (5-FU/epirubicin/cyclophosphamide), CMF (cyclophosphamide/methotrexate/5-fluorouracil) and AC (doxorubicin (adriamycin)/ cyclophosphamide) regimens for breast cancer. The repair of DNA adducts caused by 5-FU, cyclophosphamide and etoposide depends on HR [24-26] although this dependency on HR is only partial for the repair of cyclophosphamide-induced DNA interstrand crosslinks [27]. The decrease in cell survival that correlated with levels of RAD21 in breast cancer cells after RAD21 knockdown, is, therefore, in keeping with the dependence of breast cancer cells on the HR pathway to repair DNA damage from chemotherapy [10]. Consistent with this proposition, we noted that both topoisomerase II and the RAD21 loading protein NIPBL, showed strong coordinately regulated expression with RAD21 in breast cancer cell lines. RAD21 is recruited, in a manner dependent on the cohesin loading protein NIPBL, to the sites of DNA double strand breaks such as those generated by topoisomerase II, promoting DNA repair in human cells [28]. Further, topoisomerase II also decatenates chromosomes before the condensation needed for RAD21 cohesin-mediated chromosome segregation. Collectively, ours and other data highlight the likely effects of the DNA repair functions of RAD21 in tumor biology, and their potential importance in cancer treatment, although other aberrant 
functions of RAD21 could theoretically contribute to our observed associations and phenotypes.

In conclusion, the predictive function of RAD21 expression for chemotherapy outcome suggests that RAD21 expression could be used to guide treatment selection. For example, in high grade tumors with enhanced RAD21 expression, consideration could be given to using chemotherapeutic drugs that are inhibitors of HR repair [25]. Furthermore, it could guide the use of alternative strategies, such as drug dosage intensification, other chemotherapy drugs with activity in breast cancer, or chemotherapy in combination with radiotherapy (RT) which is a potent inducer of DNA double stand breaks. Further testing of the sensitivity of RAD21 knockdown clones to other chemotherapeutic agents may be of utility. Because of its effect on prognosis and therapeutic outcome, enhanced RAD21 expression may also be a novel therapeutic target. Many of the methodologies used to clinically counteract oncogene expression, (for example, gene therapy, antisense oligonucleotide therapy, specific microRNA expression), could be entertained to reduce RAD21 levels.

\section{Conclusions}

In summary, expression of RAD21 in a significant subset of breast cancers confers poor prognosis in high grade luminal, basal and HER2 breast cancers, and resistance to chemotherapy in breast cancer. RAD21 may be a novel marker of poor prognosis, a predictive factor for systemic therapy outcomes and a new target for breast cancer therapy.

\section{Additional material}

Additional file 1: Summary of breast cancer patient samples used for the study. A pdf file containing a table that summarizes breast cancer patient samples used for this study.

Additional file 2: Anti-RAD21 antibody validation. A pdf file showing the validation of the anti-RAD21 antibody using siRNA-mediated knockdown of the human RAD21 gene in MCF10A cells.

\begin{abstract}
Abbreviations
5-FU: 5-fluorouracil; AC: doxorubicin (adriamycin)/cyclophosphamide; CGH: comparative genomic hybridization; CMF: cyclophosphamide/methotrexate/ 5-fluorouracil; DAB: 3': 3'-diaminobenzidine; DCIS: ductal carcinoma in situ; DMEM: Dulbecco's modified Eagle medium; EGF: epidermal growth factor; HR: homologous recombination; HRP: horseradish peroxidase; HRR: homologous recombinational repair; PC2: Physical Containment level 2; PGK: phosphoglycerate kinase; qRT-PCR: quantitative real time PCR; RFS: relapsefree survival; RMA: Robust Multichip Analysis; RT: radiotherapy; SCC: sister chromatid cohesion; shRNA: short-hairpin RNA; siRNA: small interference RNA; SNP: single nucleotide polymorphism; TMA: tissue microarray.
\end{abstract}

\section{Acknowledgements}

This study was supported in part by the Victorian Breast Cancer Research Consortium, the Victorian Cancer Biobank, Cancer Council Victoria (Grant 091224 to HX and MJM), Cancer Institute of New South Wales, the
Australian Cancer Research Foundation, the Petre Foundation, the National Health and Medical Research Council (Project grant 400207 to HX; Project Grants: 535903 and 535947 to EAKM and RLS) and the RT Hall Trust, Australia. SS is supported by grants from the Netherlands Genomics Initiative (NGI)/NWO.

\section{Author details}

${ }^{1}$ Research Division, Peter MacCallum Cancer Centre, Locked Bag 1, A'Beckett Street, Melbourne, Vic 8006, Australia. ²Department of Pathology, Peter MacCallum Cancer Centre, Locked Bag 1, A'Beckett Street, Melbourne, Vic 8006, Australia. ${ }^{3}$ Department of Pathology, Faculty of Medicine and Dental Sciences, The University of Melbourne, Elizabeth Street, Parkville, Vic 3000, Australia. ${ }^{4}$ The Breakthrough Breast Cancer Research Centre, Institute of Cancer Research, 237 Fulhan Road, London SW3 6JB, UK. ${ }^{5}$ Department of Bioinformatics, Erasmus University Medical Centre, Dr. Molewaterplein 50, 3015 GE Rotterdam, The Netherlands. 'Department of Genetics, Erasmus University Medical Centre, and Cancer Genomics Centre, Dr. Molewaterplein 50, 3015 GE Rotterdam, The Netherlands. ${ }^{7}$ Department of Radiation Oncology, Peter MacCallum Cancer Centre, Locked Bag 1, A'Beckett Street, Melbourne, Vic 8006, Australia. ${ }^{8}$ Cancer Research Program, Garvan Institute of Medical Research, 384 Victoria Street, Darlinghurst, NSW 2010, Australia. ${ }^{9}$ Department of Anatomical Pathology, South Eastern Area Laboratory Service, St George Hospital, Gary Street, Kogarah, NSW 2217, Australia. ${ }^{10} \mathrm{School}$ of Medical Sciences, University of NSW, High Street, Kensington, NSW 2052, Australia. ${ }^{11}$ School of Medicine, University of Western Sydney, Locked Bag 1797, Penrith, NSW 2751, Australia. ${ }^{12}$ St Vincent's Clinical School, University of NSW, Victoria Street, Darlinghurst, NSW 2010, Australia. ${ }^{13}$ Department of Tissue Pathology and Diagnostic Oncology, Royal Prince Alfred Hospital, Missenden Road, Camperdown, NSW 2050, Australia.

${ }^{14}$ Central Clinical School, Sydney Medical School, Edward Ford Building A27, The University of Sydney, NSW 2006, Australia. ${ }^{15}$ Department of Medical Oncology, Westmead Hospital, Darcy Road, Westmead, NSW 2145, Australia. ${ }^{16}$ Breast Cancer Institute, Westmead Hospital, Darcy Road, Westmead, NSW 2145, Australia. ${ }^{17}$ Western Clinical School, Sydney Medical School, Edward Ford Building A27, The University of Sydney, NSW 2006, Australia.

${ }^{18}$ Australian National University and Department of Radiation Oncology, Canberra Hospital, Yamba Drive, Garran, Australian Capital Territory 2605, Australia.

\section{Authors' contributions}

HX contributed to the conception and design of the study, performed experiments, data analysis and interpretation, and drafted and revised the manuscript. MY scored and analyzed immunohistochemistry data, performed the statistical analysis, and drafted and revised the manuscript. JP participated in antibody validation, immuno-staining and the generation of RAD21 knockdown cells. RN contributed to the collection, analysis and interpretation of $\mathrm{CGH}$ and microarray data, and revised the manuscript. YY participated in immuno-staining, characterization and survival assays of RAD21 knockdown cells. SS and PS carried out the analysis and interpretation of microarray data on cell lines, and revised the manuscript. JMT participated in the gene expression analysis, and revised the manuscript. SV performed qRT-PCR analysis and contributed to data analysis. JSRF contributed to CGH and microarray data analysis and interpretation. EAKM, SAO, CMN and RLS contributed to the study materials, patient data collection, and revised the manuscript. RGR contributed to the conception and design, data analysis and interpretation, and revised the manuscript. MJM contributed to the conception and design of the study, data analysis and interpretation, and drafted and revised the manuscript. SF contributed to the study conception and design, provision of study materials, collection and assembly of data, data analysis and interpretation, and drafted and revised the manuscript. All authors read and approved the final manuscript.

\section{Competing interests}

The authors declare that they have no competing interests.

Received: 16 July 2010 Revised: 11 August 2010

Accepted: 21 January 2011 Published: 21 January 2011

\section{References}

1. McKay MJ, Troelstra C, van der Spek P, Kanaar R, Smit B, Hagemeijer A, Bootsma D, Hoeijmakers $\mathrm{JH}$ : Sequence conservation of the rad21 
Schizosaccharomyces pombe DNA double-strand break repair gene in human and mouse. Genomics 1996, 36:305-315.

2. Nasmyth K: Disseminating the genome: joining, resolving, and separating sister chromatids during mitosis and meiosis. Annu Rev Genet 2001, 35:673-745.

3. Peters JM, Tedeschi A, Schmitz J: The cohesin complex and its roles in chromosome biology. Genes Dev 2008, 22:3089-3114.

4. Gause M, Schaaf CA, Dorsett D: Cohesin and CTCF: cooperating to control chromosome conformation? Bioessays 2008, 30:715-718.

5. Birkenbihl RP, Subramani S: Cloning and characterization of rad21 an essential gene of Schizosaccharomyces pombe involved in DNA doublestrand-break repair. Nucleic Acids Res 1992, 20:6605-6611.

6. Parelho V, Hadjur S, Spivakov M, Leleu M, Sauer S, Gregson HC, Jarmuz A, Canzonetta C, Webster Z, Nesterova T, Cobb BS, Yokomori K, Dillon N, Aragon L, Fisher AG, Merkenschlager M: Cohesins functionally associate with CTCF on mammalian chromosome arms. Cell 2008, 132:422-433.

7. Wendt KS, Peters JM: How cohesin and CTCF cooperate in regulating gene expression. Chromosome Res 2009, 17:201-214.

8. Nasmyth K, Haering CH: Cohesin: Its Roles and Mechanisms. Annu Rev Genet 2009, 43:525-558.

9. Rhodes DR, Yu J, Shanker K, Deshpande N, Varambally R, Ghosh D, Barrette T, Pandey A, Chinnaiyan AM: Large-scale meta-analysis of cancer microarray data identifies common transcriptional profiles of neoplastic transformation and progression. Proc Natl Acad Sci USA 2004, 101:9309-9314.

10. Atienza JM, Roth RB, Rosette C, Smylie KJ, Kammerer S, Rehbock J, Ekblom J, Denissenko MF: Suppression of RAD21 gene expression decreases cell growth and enhances cytotoxicity of etoposide and bleomycin in human breast cancer cells. Mol Cancer Ther 2005, 4:361-368.

11. Porkka KP, Tammela TL, Vessella RL, Visakorpi T: RAD21 and KIAA0196 at $8 q 24$ are amplified and overexpressed in prostate cancer. Genes Chromosomes Cancer 2004, 39:1-10.

12. van't Veer $L J$, Dai H, van de Vijver MJ, He YD, Hart AA, Mao M, Peterse HL, van der Kooy K, Marton MJ, Witteveen AT, Schreiber GJ, Kerkhoven RM, Roberts C, Linsley PS, Bernards R, Friend SH: Gene expression profiling predicts clinical outcome of breast cancer. Nature 2002, 415:530-536.

13. McShane LM, Altman DG, Sauerbrei W, Taube SE, Gion M, Clark GM: Reporting recommendations for tumor marker prognostic studies (REMARK). J Natl Cancer Inst 2005, 97:1180-1184.

14. Yan M, Rayoo M, Takano EA, Fox SB: BRCA1 tumours correlate with a HIF1alpha phenotype and have a poor prognosis through modulation of hydroxylase enzyme profile expression. $\mathrm{Br} J$ Cancer 2009, 101:1168-1174.

15. Nielsen TO, Hsu FD, Jensen K, Cheang M, Karaca G, Hu Z, HernandezBoussard T, Livasy C, Cowan D, Dressler L, Akslen LA, Ragaz J, Gown AM, Gilks CB, van de Rijn M, Perou CM: Immunohistochemical and clinical characterization of the basal-like subtype of invasive breast carcinoma. Clin Cancer Res 2004, 10:5367-5374.

16. Livak KJ, Schmittgen TD: Analysis of relative gene expression data using real-time quantitative PCR and the 2(-Delta Delta C(T)) Method. Methods 2001, 25:402-408.

17. Xu H, Beasley M, Verschoor S, Inselman A, Handel MA, McKay MJ: A new role for the mitotic RAD21/SCC1 cohesin in meiotic chromosome cohesion and segregation in the mouse. EMBO Rep 2004, 5:378-384.

18. Natrajan R, Weigelt B, Mackay A, Geyer FC, Grigoriadis A, Tan DS, Jones C, Lord CJ, Vatcheva R, Rodriguez-Pinilla SM, Palacios J, Ashworth A, ReisFilho JS: An integrative genomic and transcriptomic analysis reveals molecular pathways and networks regulated by copy number aberrations in basal-like, HER2 and luminal cancers. Breast Cancer Res Treat 2010, 121:575-89.

19. Hollestelle A, Nagel JH, Smid M, Lam S, Elstrodt F, Wasielewski M, Ng SS, French PJ, Peeters JK, Rozendaal MJ, Riaz M, Koopman DG, Ten Hagen TL, de Leeuw BH, Zwarthoff EC, Teunisse A, van der Spek PJ, Klijn JG, Dinjens WN, Ethier SP, Clevers H, Jochemsen AG, den Bakker MA, Foekens JA, Martens JW, Schutte M: Distinct gene mutation profiles among luminal-type and basal-type breast cancer cell lines. Breast Cancer Res Treat 2010, 121:53-64.

20. GEO. [http://www.ncbi.nlm.nih.gov/geo/].

21. Franken NA, Rodermond HM, Stap J, Haveman J, van Bree C: Clonogenic assay of cells in vitro. Nat Protoc 2006, 1:2315-2319.
22. Richardson C, Stark JM, Ommundsen M, Jasin M: Rad51 overexpression promotes alternative double-strand break repair pathways and genome instability. Oncogene 2004, 23:546-553.

23. Husain A, He G, Venkatraman ES, Spriggs DR: BRCA1 up-regulation is associated with repair-mediated resistance to cisdiamminedichloroplatinum(II). Cancer Res 1998, 58:1120-1123.

24. Matuo R, Sousa FG, Escargueil AE, Soares DG, Grivicich I, Saffi J, Larsen AK, Henriques JA: DNA repair pathways involved in repair of lesions induced by 5 -fluorouracil and its active metabolite FdUMP. Biochem Pharmacol 2010, 79:147-153.

25. Helleday T, Petermann E, Lundin C, Hodgson B, Sharma RA: DNA repair pathways as targets for cancer therapy. Nat Rev Cancer 2008, 8:193-204.

26. Treszezamsky AD, Kachnic LA, Feng Z, Zhang J, Tokadjian C, Powell SN: BRCA1- and BRCA2-deficient cells are sensitive to etoposide-induced DNA double-strand breaks via topoisomerase II. Cancer Res 2007, 67:7078-7081.

27. Vasquez KM: Targeting and processing of site-specific DNA interstrand crosslinks. Environ Mol Mutagen 2010, 51:527-539.

28. Bauerschmidt C, Arrichiello C, Burdak-Rothkamm S, Woodcock M, Hill MA, Stevens DL, Rothkamm K: Cohesin promotes the repair of ionizing radiation-induced DNA double-strand breaks in replicated chromatin. Nucleic Acids Res 2010, 38:477-487.

doi:10.1186/bcr2814

Cite this article as: Xu et al:: Enhanced RAD21 cohesin expression confers poor prognosis and resistance to chemotherapy in high grade luminal, basal and HER2 breast cancers. Breast Cancer Research 2011 13: R9.

\section{Submit your next manuscript to BioMed Central and take full advantage of:}

- Convenient online submission

- Thorough peer review

- No space constraints or color figure charges

- Immediate publication on acceptance

- Inclusion in PubMed, CAS, Scopus and Google Scholar

- Research which is freely available for redistribution

Submit your manuscript at www.biomedcentral.com/submit
C) Biomed Central 


\section{University Library}

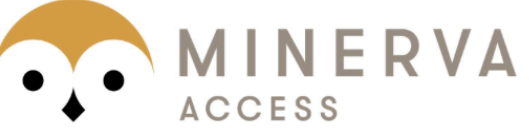

A gateway to Melbourne's research publications

Minerva Access is the Institutional Repository of The University of Melbourne

Author/s:

Xu, H;Yan, M;Patra, J;Natrajan, R;Yan, Y;Swagemakers, S;Tomaszewski, JM;Verschoor, S;Millar, EKA;van der Spek, P;Reis-Filho, JS;Ramsay, RG;O'Toole, SA;McNeil, CM;Sutherland, RL;Mckay, MJ;Fox, SB

Title:

Enhanced RAD21 cohesin expression confers poor prognosis and resistance to chemotherapy in high grade luminal, basal and HER2 breast cancers

Date:

2011-01-01

Citation:

Xu, H., Yan, M., Patra, J., Natrajan, R., Yan, Y., Swagemakers, S., Tomaszewski, J. M., Verschoor, S., Millar, E. K. A., van der Spek, P., Reis-Filho, J. S., Ramsay, R. G., O'Toole, S. A., McNeil, C. M., Sutherland, R. L., Mckay, M. J. \& Fox, S. B. (2011). Enhanced RAD21 cohesin expression confers poor prognosis and resistance to chemotherapy in high grade luminal, basal and HER2 breast cancers. BREAST CANCER RESEARCH, 13 (1), https:// doi.org/10.1186/bcr2814.

Persistent Link:

http://hdl.handle.net/11343/264777

License:

CC BY 